REVISTA ANDALUZA DE ANTROPOLOGÍA

NÚMERO 18: MIRADAS DESDE LA ANTROPOLOGÍA AMBIENTAL.

ABRIL DE 2020

ISSN 2174-6796

[pp. 31-47]

https://dx.doi.org/10.12795/RAA.2020.18.03

\title{
LA AGRICULTURA ECOLÓGICA COMO "ANHELO". REFLEXIONES SOBRE POLISEMIA Y SOSTENIBILIDAD
}

\author{
Ernesto Martínez Fernández Correo \\ Universidad Pablo de Olavide
}

\section{Resumen}

Las aproximaciones antropológicas a la agricultura ecológica se han venido centrando en la agricultura ecológica certificada. En este artículo de reflexión conceptual, se hace un recorrido por los orígenes y trayectoria de ésta, así como se profundiza en el estado de la cuestión de los abordajes antropológicos sobre la temática. A partir de ahí, se intenta trascender aquella focalización en la agricultura ecológica en cuanto objeto de estudio identificado en exclusiva con una modalidad de manejo agrario. En ese sentido, se indaga en otro nivel de abstracción, aquél en el que la agricultura ecológica aparece como una idea cargada de magnetismo simbólico. Para ello, el artículo se interroga sobre los vínculos entre el proceso de normalización experimentado por el discurso ambiental, el concepto de sostenibilidad y el de agricultura ecológica.

Palabras clave: agricultura ecológica, antropología, sostenibilidad, anhelo social.

\section{Abstract}

Anthropological engagements with organic farming have focused on certified organic farming up to now. This article describes the origin and evolution of that style of farming, as well as offers a review of the anthropological approaches to the topic. Taking these issues into account, it attempts to go beyond that identification. In that sense, another level of abstraction is explored: that of organic farming as an idea filled with symbolic 
magnetism. To that end, the article examines the historical and symbolic ties among the process of normalisation of the environmental discourse, the concept of sustainability and organic farming.

Keywords: organic farming, anthropology, sustainability, social longing

\section{INTRODUCCIÓN ${ }^{1}$}

La expresión "agricultura ecológica” está cada vez más presente en nuestras vidas. Por los barrios de las ciudades no dejan de aparecer pequeños comercios de productos ecológicos. Entre los supermercados, pocos quedan que no dediquen en exclusiva alguna de sus calles o estanterías a este tipo de alimentos. En la televisión y en internet, pululan anuncios que utilizan la agricultura ecológica como gancho publicitario. También en la calle es frecuente toparse con este tipo de propaganda e, incluso, con otras tipologías de reclamos "ecológicos" 2 .

Buena parte de la relevancia social de la que estos ejemplos impresionistas son indicio tiene que ver con el desarrollo que en las últimas décadas ha experimentado la modalidad de manejo agrario que se ha dado en llamar "agricultura ecológica certificada". Podría decirse que ésta constituye una versión simplificada de las agriculturas alternativas que surgieron durante los dos primeros tercios del siglo XX en Europa (Alemania, Reino Unido, Francia, Suiza $)^{3}$ y consiste, a grandes rasgos, en una agricultura de sustitución de los insumos convencionales por otros ecológicos sometida a procesos de auditoría institucionalizados.

La razón de la mencionada simplificación habría que buscarla en el crecimiento que, hacia 1970, se estaba produciendo tanto en el número de agricultores ecológicos como

1. La investigación con la que se vincula esta publicación fue realizada en el marco de mi tesis doctoral (Ponerse verde. Ecologización y economía moral entre los pequeños ganaderos de la Sierra Morena Sevillana, 2019), financiada mediante una beca del programa de Formación de Profesorado Universitario (FPU) del antiguo Ministerio de Educación, Cultura y Deporte.

2. Un ejemplo curioso lo encontraba no hace mucho, cuando me topaba con un cartel que interpelaba al viandante a través del siguiente mensaje: “Tenga su propio huerto ecológico". Sorprendentemente, la adquisición a la que se invita no consiste en la compra de un terreno, sino del derecho a su planificación hortícola, esto es, lo que se adquiriría sería el derecho al diseño del huerto desde la mesa del ordenador, sin necesidad de participar en cultivo alguno. De esto último se encargaría, supuestamente, el verdadero dueño del terreno.

3. Ver Guzmán, González de Molina y Sevilla (2000) y Vogt (2007) para profundizar en esas modalidades previas de agricultura ecológica. 
en la demanda de sus productos. Ello derivó en el uso de etiquetas propias y, asociado con ello, en la redacción por parte de las asociaciones de agricultores ecológicos de normas donde se recogían los estándares y requisitos mínimos que debían seguir sus miembros. La británica Soil Association publicaba sus normas ya en 1967, mientras que la francesa Nature et Progrès lo hacía en 1972. En 1976, comenzaba en Suiza el debate y redacción de unos estándares de ámbito nacional (Schmid, 2007: 154). Este último paso fue fundamental para que poco tiempo después la Federación Internacional de Movimientos de Agricultura Ecológica (IFOAM, en sus siglas en inglés) se plantease la formulación de unas normas internacionales, dado que "una tarea urgente consistía en dar una definición más precisa a lo que se quería decir con los términos 'agricultura biológica', 'orgánica', 'ecológica' y 'natural', y recopilar un conjunto común de estándares para los productos vendidos bajo tales etiquetas o garantías" (ibid.: 155). Las "normas básicas" de IFOAM fueron publicadas en 1980 y hasta la actualidad son objeto de revisión periódica (Dankers, 2004: 15).

La aparición de unos estándares que tenían como finalidad generar una suerte de consenso de mínimos derivó en un relajamiento de la mirada holística que caracterizaba al conjunto de modalidades ecológicas previas a la "certificada". A ello contribuyó también una creciente orientación hacia el mercado y, concretamente, hacia un nicho de consumidores urbanos de elevado poder adquisitivo cuya principal demanda era la de productos sanos frente al contenido en agrotóxicos de los productos de la agricultura convencional. Este elemento es clave para entender la progresiva transformación en una agricultura de sustitución de los insumos químicos sintéticos por otros "naturales". Por ese camino, la agricultura ecológica (certificada) ha pasado a depender fuertemente de la agroindustria, tanto en lo que tiene que ver con los insumos (Guzmán, González de Molina y Sevilla, 2000: 69-70) como con la distribución de sus productos (Constance, 2018: 6). "Convencionalización" ha sido el término mediante el que varios autores han conceptualizado este proceso (Buck, Getz y Guthman, 1997; Guthman, 2004).

Gracias en buena parte a esta absorción y al apoyo económico vía ayudas estatales de que goza en un número creciente de territorios -el caso de la Unión Europea (UE) quizás sea el más destacado-, la agricultura ecológica certificada es actualmente un fenómeno en claro crecimiento a escala global. Si en vísperas del tercer milenio la superficie mundial era de 11 millones de hectáreas ecológicas certificadas (1999), esa cifra casi se septublicaba veinte años después, alcanzando en 2018 los 71,5 millones de hectáreas (Schlatter, Trávníček, Lernoud y Willer, 2020: 37).

4. La pérdida de esa perspectiva holística tiene que ver con el no desarrollo de "mecanismos de autorregulación del sistema (alta biodiversidad, infraestructura ecológica: setos, etc.), ni de conservación de los recursos (agua, suelo, etc.). Así, graves problemas de la agricultura mundial como la erosión del suelo, la salinización y alcalinización del mismo provocada por el mal uso del agua de riego, y otros procesos de degradación de los recursos naturales no son considerados" (Guzmán et al., 2000: 69). 
Como veremos en el próximo apartado, las aproximaciones antropológicas a la agricultura ecológica se han centrado casi exclusivamente en los efectos asociados al fenómeno de la agricultura ecológica certificada. La cuestión reside en que dicho objeto no agota todas las posibilidades de la agricultura ecológica en cuanto a su significación social.. Este artículo, de reflexión conceptual, intenta precisamente ir más allá del sentido de agricultura ecológica como estilo de manejo e indagar en otro nivel de abstracción, aquél en el que la agricultura ecológica aparece como una idea cargada de magnetismo simbólico. Para ello, se interroga sobre los vínculos entre el proceso de normalización experimentado por el discurso ambiental (Santamarina, 2006), el concepto de sostenibilidad y el de agricultura ecológica.

\section{ANTROPOLOGÍA Y AGRICULTURA ECOLÓGICA}

La Antropología no ha sido ajena a la relevancia social adquirida en los últimos lustros por la agricultura ecológica en casi todos los rincones del planeta. En algunos casos, el interés antropológico ha sido más o menos tangencial, como pone de manifiesto el caso de West (2010), quien, siguiendo la crítica foucaultiana de Guthman $(2007,2008)$ a las "etiquetas de calidad", se adentra en la cadena que lleva el café etiquetado como ecológico y "de comercio justo" desde Papúa Nueva Guinea hasta Estados Unidos. Si bien realiza trabajo de campo en Papúa para conocer las condiciones reales de producción de ese café, el autor concentra su atención sobre la "producción del consumidor" de este particular mercado.

Otras investigaciones, realizadas plenamente en ámbitos agrarios, se han interesado por la agricultura ecológica como parte de la "reconversión" experimentada por el medio rural de los países del continente europeo. En el contexto de la ampliación de la UE a los países del Este, Fox (2011) aborda las relaciones del campesinado rumano con la implementación de la Política Agrícola Común (PAC). Parte de su estudio se centra en los efectos mercantilizadores sobre la producción campesina de la creación de marcas y distintivos ecológicos certificados, pero también en las "maniobras" practicadas por esos grupos domésticos en su particular adaptación al nuevo escenario.

Igualmente interesada por la agricultura ecológica como vector de cambio del medio rural europeo encontramos la tesis de Lozano (2011), centrada en el análisis de las estrategias económicas de los productores ecológicos de la comarca andaluza de la Sierra de Segura. Los esfuerzos de esta autora se dirigen al estudio de las cooperativas olivareras ecológicas, la promoción del sector y las relaciones con diferentes organismos estatales con el fin de demostrar la aparición de un "sistema agroalimentario localizado"5 ecológico. Asimismo, la autora realiza un perfil de los agricultores ecológicos de la comarca, dividiéndolos en dos colectivos vinculados a la pertenencia a una u otra de las cooperativas ecológicas

5. Para profundizar en este concepto, ver Fournier y Muchnik (2012). 
locales: uno formado por agricultores a tiempo completo y caracterizado por desarrollar una agricultura de sustitución de insumos; otro por una perspectiva más holística y el predominio de la dedicación a tiempo parcial ${ }^{6}$.

Lozano $(2011,2013)$, al objeto de entender el "estancamiento" del sistema agroalimentario localizado que habría verificado durante su trabajo de campo, también explora las diferencias a nivel de representaciones locales sobre la agricultura ecológica entre los agricultores convencionales y ecológicos, dibujando un conflicto más o menos latente entre dos colectivos que entienden sus prácticas agrarias como antagónicas. Debe citarse, al hilo de esta contraposición, el estudio de Hetherington (2005), que profundiza sobre las relaciones entre agricultores convencionales y ecológicos en la provincia canadiense de Nueva Escocia.

Desde una perspectiva alejada de la problemática aplicada del "desarrollo rural" -pero no del desarrollo rural como objeto-, Galvin (2014) se interesa por los programas institucionales de fomento de la agricultura ecológica en cuanto formas de intervención estatal (Scott, 1998) y, en consecuencia, como vectores de (re)estructuración de las relaciones entre poblaciones locales y actores estatales. A partir de una investigación realizada en la región de Uttarakhand -autodenominada "capital ecológica de la India"-, la autora describe cómo las nuevas relaciones sociales con extensionistas, auditores, etc. que lleva aparejada la certificación ecológica institucionalizada implican el desarrollo de nuevos ejes discursivos que discriminarían entre prácticas agrarias objeto de reconocimiento y prácticas no merecedoras de ese tratamiento. Un ejemplo sería la contraposición entre las expresiones "ecológico por defecto" y "ecológico a propósito". Mientras la primera sería usada por los técnicos para categorizar las prácticas sostenibles "tradicionales" como una suerte de adaptaciones pasivas al medio, la segunda sería un medio de "reconocimiento estatal" por el que las nuevas prácticas ecológicas -mayoritariamente adoptadas por los agricultores acomodados- se entenderían como fruto de la agencia y la creatividad individual. Tal diferenciación sería aprovechada por las castas más elevadas para estrechar sus lazos con el Estado, contribuyendo de este modo el programa al reforzamiento de la desigualitaria estructura social (Galvin, 2014).

En un artículo más reciente, Galvin (2018) sitúa el foco sobre el proceso de certificación ecológica a partir de material producido en el mismo contexto etnográfico con el fin de posicionarse en el debate más amplio sobre la cuestión de la transparencia y las prácticas de auditoría. La autora se centra en los efectos derivados de la incertidumbre experimentada por los certificadores, dado el abultado número de explotaciones, la imposibilidad de verificar todas las prácticas agrarias y la incompletitud de los "diarios"

6. También Del Campo (2000) se interesa por los diferentes perfiles de productor ecológico, si bien a partir de una investigación que refiere al conjunto andaluz. 
en que los agricultores deben describir las mismas. Aunque, por un lado, ello resulta en un impulso al refinamiento de la práctica auditora -vía introducción de análisis de residuos-, también habría derivado en la generalización entre los auditores de una ideología de la confianza (viśvās) en relación a los agricultores. Esta ideología, con su retórica del compromiso, la honestidad y el honor, sería necesaria para sostener el edificio de la certificación ante la confirmación de que, en la práctica, transparencia y opacidad se engendran mutuamente.

\section{EN PLURAL}

En la recapitulación del apartado anterior puede apreciarse cómo las distintas aproximaciones antropológicas al fenómeno de la agricultura ecológica lo han hecho centrando su atención sobre la modalidad de manejo que aquí se ha dado en llamar agricultura ecológica certificada. Sin embargo, cabe preguntarse si la agricultura ecológica significa algo más; si, como apuntan Campbell y Liepins (2001), las formas de entender la agricultura ecológica trascienden las prácticas agrarias para constituir todo un campo discursivo.

Valga una viñeta extraída de mi trabajo de campo doctoral entre los ganaderos de la Sierra Morena Sevillana para ilustrar esa pluralidad de sentidos. Durante mi investigación, seguí muy de cerca a una pareja de ganaderos. Raimundo y Carlos, padre e hijo respectivamente, tenían una pequeña explotación certificada en ecológico. Fui su "ayudante" por un tiempo y, durante el resto de la investigación, nunca dejé de visitarlos. En uno de nuestros últimos encuentros, se encontraba por allí otro visitante asiduo, un "neorrurral" de origen gallego llamado Brais. Tras acompañar a Carlos a una parcela cercana para enseñarme unos brotes de raigrás italiano7, volvimos junto a Brais y al anciano ganadero, Raimundo. Yo venía preguntándole a Carlos si el cultivo de raigrás era susceptible de certificación ecológica y, de ahí, partió la siguiente conversación:

"Mientras volvíamos, me surgió la duda de si el raigrás se podía certificar. Era algo que no había preguntado aún ni recordaba [de mis lecturas sobre normativa ecológica]. Carlos respondió sorprendido que sí: "Claro, ¿por qué no? Si nosotros lo curtivamos iguá que la arfarfa, la avena...". Ya muy cerca de Raimundo y Brais, Carlos añadía: "Pero, en fin, si pudiera vorvé atrás, no me metería en la agricurtura ecológica". Subió el tono: "Y, además, no creo en er bombo de lo ecológico". Carlos parecía aprovechar la presencia de Brais, un esperable defensor de la agricultura ecológica, para dejar clara su posición. Quizás no. Brais no hace amago de dar su opinión. Si el amago era parsimonia, tampoco Raimundo le permite mucho tiempo y entra por la brecha

7. El raigrás italiano (Lolium multiflorum) es una planta perteneciente a la familia de las gramíneas que Raimundo y Carlos cultivaban para servir como alimento para sus ovejas. 
abierta por su hijo: "Los ecologistas deberían está tos [todos] muertos, porque son los que se están cargando to esto". El anciano acompaña su intervención de un gesto abarcante con el brazo y una mirada al horizonte para dar a entender que con "to esto" se refiere al agro local. Yo intento hurgar en la indignación de Raimundo y le pregunto si, entonces, su explotación sería ecológica. Llamativamente, me responde que sí, argumentando que 'nosotros no echamos na". Carlos añade: "Somos ecológicos porque seguimos hasiendo to como antiguamente" (Diario de campo, diciembre de 2016)"s.

A botepronto, puede resultar tremendamente sorprendente la oscilación en apenas segundos con respecto a lo que estos pequeños ganaderos entendían por "agricultura ecológica”. Una mirada superficial concluiría que ambos estaban diciendo una cosa y la inversa casi al mismo tiempo. Muchos incluso correrían a etiquetar a Raimundo y Carlos como una suerte de personajes incongruentes, incapaces, quizás, de entender el verdadero "significado" de la agricultura ecológica. Una mirada desde la empatía etnográfica, por el contrario, se preguntaría si esa aparente incoherencia lo es en efecto. En ese sentido, podría ser interesante mirar a las intervenciones de estos ganaderos a partir de la noción de "espacio de discursos". Para Martín (2014), un espacio de discursos tiene que ver con el conjunto de discursos y sentidos a disposición de las personas en relación a un determinado asunto. En las interacciones discursivas, los argumentos se desarrollarían en función de esos discursos disponibles. Según este autor, "todo argumento es un contraargumento" (ibid.: 131).

Si adoptamos esta perspectiva, da la impresión de que el primer sentido frente al que Carlos parecía posicionarse era el de la agricultura ecológica entendida como una modalidad de manejo delimitada por las administraciones agrarias y apadrinada por éstas vía ayudas. Ello es evidente en su afirmación "no creo en er bombo de lo ecológico", que apunta al apoyo institucional y mediático ("bombo") del que goza esa agricultura ecológica. Raimundo, aprovechando las palabras de su hijo, centraba su intervención en el nivel productivo. Indignado, afirmaba: "Los ecologistas deberían está tos muertos, porque son los que se están cargando to esto". Hay que puntualizar que con el término "ecologistas" englobaba a distintos personajes ligados con un pensamiento o práctica ambientalista y/o conservacionista (trabajadores del Parque Natural implementado en la comarca, activistas, políticos, etc). Como en otras zonas rurales de Andalucía (Coca,

8. En la transcripción de la modalidad lingüística hablada por mis interlocutores se ha buscado un equilibrio entre la visibilización de sus características y la evitación de textos de complicada lectura. La regla seguida ha sido la de no explicitar aquellos rasgos que implicarían la inclusión de letras o signos ortográficos ajenos a la escritura estandarizada del castellano. Aprovecho para aclarar que los nombres de los interlocutores que aparecen en el apartado son seudónimos. 
2008; Cortés, 2012), en la Sierra Morena Sevillana existe una profunda insatisfacción respecto al declive general de la actividad agraria y algunas de las críticas más usuales ven complicidad -cuando no responsabilidad directa- en las distintas políticas y actores (agro)ambientales que operan en estos territorios.

CarlosyRaimundohabían cargado contra doselementosquecaracterizaríanala agricultura ecológica entendida como una modalidad de manejo avalada institucionalmente. Ahora bien, mi introducción de la pregunta “¿vuestra explotasión sería ecológica?" parece que los llevaba a entender que el sentido frente al que posicionarse cambiaba. En ese sentido, ambos ganaderos pasaban con soltura a otro registro y se reconocían sin dudarlo como "ecológicos". Raimundo lo argumentaba a través de su manejo no contaminante: "no echamos na"; Carlos, aludiendo a una cierta noción de tradicionalidad: "seguimos hasiendo to como antiguamente".

A mi juicio, este segundo sentido de la agricultura ecológica tiene que ver con otro nivel de abstracción, con una idea de agricultura que se entiende como vinculada con aquella "agricultura de toda la vida" que resistió el embate de la llamada revolución verde, pero al mismo tiempo absolutamente contemporánea y caracterizada por una forma de manejo no contaminante y sostenible. Subrayo sostenible porque considero que este segundo sentido de la agricultura ecológica, ejemplificado aquí a través del caso de Raimundo y Carlos, no puede entenderse sin atender al proceso de normalización de que ha sido objeto la crítica ecologista desde que emergiese con vigor en los años sesenta del siglo pasado. Ese proceso ha arrastrado consigo al anverso propositivo de la preocupación ambiental, que, como se argumentará, quedó recogido en el concepto de sostenibilidad. El esclarecimiento de los lazos semánticos entre medio ambiente, sostenibilidad y agricultura ecológica es el objeto del próximo apartado.

\section{SOSTENIBILIDAD Y AGRICULTURA ECOLÓGICA}

\subsection{Medio ambiente y sostenibilidad}

La aceleración industrial que siguió a la II Guerra Mundial y su posguerra no tardó en acentuar los problemas medioambientales existentes y dar lugar a otros nuevos. De entre ellos, aquél que abrió la veda para la socialización de la denuncia ecologista era el de la contaminación de suelos, aguas y atmósfera, en particular, por el peligro que ello representaba para el cuerpo humano y la salud pública. Serían libros como La primavera silenciosa (1962) o Ciencia y supervivencia (1963), de Rachel Carson y Barry Commoner respectivamente, los que conseguirían conectar con un amplio público a través de un estilo que unía análisis científicos de corte ecológico con referencias a los efectos concretos de la polución que los potenciales lectores podían comprobar a su alrededor (Santamarina, 2006: 66). En los años siguientes, muchas obras siguieron su 
estela y consiguieron convertirse también en "superventas" gracias a ese estilo alarmista y directo (ibid.: 68).

Al impacto de esas obras debe unirse la potencia de los medios de comunicación, con la televisión a la cabeza. Su capacidad para reflejar los problemas ambientales ocurridos en distintas partes del globo la llevó a ser uno de los elementos fundamentales para la extensión de la "nueva sensibilidad" ambiental. Asimismo, no puede olvidarse el clima de insatisfacción con el sistema que tuvo su principal materialización en las protestas y revueltas de 1968 (Santamarina, Vaccaro y Beltran, 2015: 15). En este contexto, y con base en determinados sectores medios urbanos, se produciría un rápido crecimiento del movimiento ecologista. En Norteamérica, el número de afiliados a las asociaciones ambientalistas ya existentes -Sierra Club, National Audubon Society- se multiplicaría casi por diez entre 1960 y 1972, mientras al mismo tiempo no dejaban de nacer organizaciones ecologistas, algunas dedicadas específicamente a cuestiones como la lucha contra la extinción de especies o la denuncia antinuclear (Santamarina, 2006: 75-76). En Europa occidental, habría que añadir la fundación de los primeros partidos verdes en países como Alemania, Bélgica o Reino Unido (Caradonna, 2014: 107).

Puede afirmarse que, a principios de la década de 1970, se había producido un importante cambio cultural consistente en la generalización de una "nueva sensibilidad" respecto al medio ambiente ${ }^{9}$. Y es que el gran logro del ecologismo fue generalizar el concepto de medio ambiente. Frente a la idea de naturaleza, entendida como una suerte de "cuerno de la abundancia" separado dicotómicamente de la sociedad humana y a su disposición (Descola, 1996/2001), el concepto de medio ambiente, como sostiene Caradonna (2014: 90), se inspiraba en las premisas de la ciencia ecológica para enfatizar la pertenencia y dependencia de los seres humanos respecto del entorno ${ }^{10}$.

9. A los factores mencionados, Stavrakakis (2000) añade otro de carácter político: la dislocación ideológica que supuso la crisis de la izquierda en ciertos países occidentales y la necesidad de reorganización del campo político transformador. Afirma este autor:

"Por razones diversas, en un momento cronológico determinado las ideologías radicales no pueden desempeñar más el trabajo de toda ideología, esto es, (...) ofrecer respuestas "creíbles", respuestas atractivas en sentido hegemónico a dislocaciones sociales que toman la forma de "enfermedades sociales" como el desempleo y la desigualdad. (...) [L] a tradición radical necesitaba una rearticulación, una nueva realización de su potencial. Esta nueva articulación fue llevada a cabo alrededor de la "naturaleza", un significante altamente catexizado [cathected: cargado (socialmente) de deseo] del discurso y la discusión pública; un significante con un potencial radical" (ibid.: 115; mi traducción). 10. Ver Cruzada (2017) y Martínez (2020) para sendas refutaciones etnográficas de la generalización y capilaridad que algunos/as antropólogos/as atribuyen al concepto de naturaleza y a la ontología naturalista (Descola 1996/2001) en contextos "occidentales". 
Como una de las derivaciones de aquel ambiente de crítica ecologista, hay que mencionar el jalón fundamental que supuso la publicación en 1972 del informe Los límites del crecimiento. El estudio fue encargado por el Club de Roma, una organización internacional de carácter filantrópico, al grupo de investigación sobre dinámica de sistemas del Instituto de Tecnología de Massachusetts (MIT, en sus siglas en inglés). Las conclusiones del informe eran categóricas: de seguir el ritmo de crecimiento económico y demográfico, en alrededor de un siglo los recursos del planeta dejarían de ser suficientes. Se hacía necesaria la implementación de una economía de "crecimiento cero" (Santamarina, 2006: 88-89). El impacto de un informe dotado de tamaña aura tecnocientífica fue fenomenal, dando lugar al fortalecimiento de la nueva disciplina en ciernes de la Economía ecológica y al llamado "debate sobre el crecimiento" (Caradonna, 2014: 105).

Fue en el seno de este debate donde se produjo la introducción de un nuevo término: sostenibilidad. En realidad, la palabra no era nueva. Hundía sus raíces en el periodo formativo de la disciplina de la silvicultura (siglos XVII-XVIII), cuya emergencia se hallaba estrechamente relacionada con la deforestación a que impulsaba el desarrollo comercial y demográfico contemporáneo. Entendiendo la deforestación como una amenaza para la independencia energética y naval de los países europeos, fueron surgiendo obras e iniciativas dedicadas a una gestión que permitiese la regeneración de sus terrenos forestales (Vehkamäki, 2005; Warde, 2018). Hay que mencionar la obra del inglés John Evelyn Sylva (1664); también, el empeño del ministro francés Jean-Baptiste Colbert por crear un "código forestal" (1669) dirigido a contrarrestar el proceso de deforestación. De la obra de ambos bebería Hans Carl von Carlowitz para redactar su tratado Sylvicultura oeconomica (1713) (Caradonna, 2014:35-36). En él es donde aparece por primera vez la palabra sostenible, al establecer como la regla de oro de la silvicultura "un uso continuo, permanente y sostenible [nachhaltende]" de los bosques (citado en Vehkamäki, 2005: 26).

Recuperada en el contexto del debate sobre el crecimiento de la década de 1970, la sostenibilidad ${ }^{11}$ partía ahora de la idea de crecimiento cero y de las premisas de la Ecología para indagar en lo que sería la transición hacia una relación humano-ambiental armoniosa. Éste es el sentido que tiene, por ejemplo, en el libro de Robert L. Stivers

11. Debe quedar claro que mi objetivo no es polemizar sobre los distintos usos de la palabra sostenibilidad, adscribirme a una suerte de definición "correcta" u operativizar el concepto. Para ello ver, entre otras posibilidades, las reflexiones de Naredo (1997/2004), González de Molina y Guzmán (2006) o Toledo (2015). Tampoco es mi intención entrar en el debate que opone sostenibilidad y sustentabilidad. Dado que en la realidad extra-académica tales términos son usados como sinónimos intercambiables y lo que me interesa es precisamente su "vida cotidiana" bajo la forma de la agricultura ecológica, no se han hecho distinciones, dándose prioridad al significante "sostenibilidad" a efectos exclusivamente prácticos. 
La sociedad sostenible: ética y crecimiento económico (1976). También en La sociedad sostenible: implicaciones para un crecimiento limitado (1977) de Dennis Clark Pirages, donde el autor abogaba, en este caso, por un crecimiento lento que permitiese caminar hacia una sociedad respetuosa con el medio (Caradonna, 2014: 124). La sostenibilidad, en este sentido, se fue afianzando como la "respuesta constructiva" al "cambio ecológico" y al desafío sistémico que el debate sobre el crecimiento ponía sobre la mesa (ibid.: 138). Podría decirse que el término emergió como la denominación del anverso propositivo de la crítica medioambiental, nucleado en torno a la idea de deseabilidad y necesidad de una sociedad humana capaz de conjurar el peligro de destrucción de nuestro hábitat a través de una relación equilibrada y respetuosa con los límites ecológicos del planeta.

En los años sucesivos, la sostenibilidad haría fortuna rápidamente. Para ello fue fundamental su particular adopción a lo largo de la década de 1980 por parte de altos diplomáticos, políticos y organismos dependientes de la ONU. Esta última había organizado ya en 1972 la Conferencia sobre el Medio Humano, celebrada en Estocolmo. Dedicada a los problemas ambientales, en ella se visualizó el disenso sobre los mismos de los países asistentes y la falta de voluntad política. No obstante, la crítica ecologista y las tesis sobre el crecimiento cero suponían una importante amenaza a las bases del sistema capitalista, y desde algunos sectores gubernamentales se entendió que debía darse una respuesta (Santamarina, 2006: 96, 103; Santamarina et al., 2015). Así, en 1980 la ONU encargaba al consejo científico de la Unión Internacional para la Conservación de la Naturaleza (UICN) la redacción de una "Estrategia de conservación mundial". Fue este documento el primero de los apadrinados por la ONU en el que apareciese la idea de sostenibilidad. Ahora bien, lo hacía, curiosamente, adosada al término desarrollo a través de un nuevo constructo: "desarrollo sostenible" (Caradonna, 2014: 141).

La cuestión no es sólo que los teóricos de sistemas, ecologistas y economistas ecológicos de los años setenta nunca hubieran tenido en mente la idea de desarrollo cuando estaban construyendo el paradigma del crecimiento cero del que partió la sostenibilidad. Si el documento de la UICN -que llevaba por título Estrategia de conservación mundial. Conservación de recursos vivos para el desarrollo sostenible- entendía el desarrollo en un sentido amplio, el mayor problema era que la idea de crecimiento, de forma más o menos inconsistente, era reintegrada (ibid.: 151-152). Y esta re-semantización se afianzaba en un nuevo informe que terminaría por dar carta de naturaleza al desarrollo sostenible: Nuestro futuro común (1987).

Para la redacción de este último, la ONU creó la Comisión Mundial sobre el Medio Ambiente y el Desarrollo (CMMAD). El informe definía el desarrollo sostenible como "el desarrollo que satisface las necesidades del presente sin comprometer la capacidad de las generaciones futuras para satisfacer sus propias necesidades" (CMMAD, 1992: 67, en Santamarina, 2006: 124). Aún manteniendo las ideas de "crisis total" y de necesidad de 
cambios drásticos de los informes técnicos y gubernamentales previos, ahora se reconocía explícitamente que el crecimiento no estaba reñido con el equilibrio ambiental e, incluso, que un crecimiento mayor era deseable (Luke, 1999: 136-137; Santamarina, 2006: 96102). Para Santamarina (2006: 124), el desarrollo sostenible se convertía así "en la nueva máscara" del discurso crecentista. Su éxito, en este sentido, debe verse como el éxito de la ideología neoliberal, que, ante el potencial subversivo de la crítica ambiental, hacía suya la idea de sostenibilidad y se proponía como el único antídoto de los problemas ambientales.

\subsection{Anhelo de sostenibilidad y agricultura ecológica}

La trayectoria de la sostenibilidad hasta la actualidad ha estado estrechamente vinculada a su asociación con el constructo del desarrollo sostenible. La generalizada adopción por gobiernos y medios de comunicación ha hecho de "lo sostenible" un término omnipresente. Como apuntan Cariño y Castorena (2016: 9): "En menos de treinta años (...), cualquier mercancía que pretende impactar el mercado es sustentable, cualquier empresa que desea limpiar su imagen es sustentable (...), toda política pública que se pretende innovadora es sustentable".

Ahora bien, el núcleo de sentido que está detrás de la sostenibilidad, su evocación de una relación con el medio caracterizada por el equilibrio socioambiental no ha desaparecido. Y no lo ha hecho porque el "prisma conceptual" (Caradonna, 2014: 90) sobre las relaciones humano-ambientales que consiguió instalar la crítica ecologista sigue vigente y también, por supuesto, la realidad de deterioro ambiental a la que respondía. Es, en ese sentido, que sostenible se identifica con el término que se aplica en nuestras sociedades a toda iniciativa relacionada con la armonía en las relaciones humanidad-ambiente. La lectura -predominantemente crecentista- que ofrece el constructo "desarrollo sostenible" no agota las posibilidades de (re)semantización de la sostenibilidad. Así, sostenible es también el adjetivo que movimientos de base ponen a sus iniciativas agroecológicas, sus ciudades en transición o proyectos comunitarios de producción de energías renovables.

Lo que esto quiere decir es que la sostenibilidad, entendida como aquel núcleo de sentido, como aquel anverso propositivo de la crítica ambiental, se ha convertido en nuestras sociedades en uno de esos elementos centrales en nuestras tramas culturales que, siguiendo a Žižek (1997/2003: 141-142), puede denominarse "anhelo social": ese tipo de denominadores de una plenitud ausente y deseada que son particularmente transversales, como la democracia, la libertad o la justicia.

Es precisamente en esa conversión de la sostenibilidad en anhelo donde encontramos el proceso clave para entender la potencia actual de la idea de agricultura ecológica. La actividad agraria era una de las puntas de lanza de los fenómenos contaminantes y de degradación asociados a la aceleración industrial de mediados del siglo XX. El modelo de 
la llamada "revolución verde" se basaba en una intensificación indiferente a los procesos y límites ecológicos que subyacen a la práctica de la agricultura. Como consecuencia de ello, graves efectos se generaron por todo el planeta -erosión y degradación del suelo, contaminación de la atmósfera y las aguas, pérdida de biodiversidad, etc.-, convirtiendo a la agricultura industrializada en uno de los factores más relevantes del proceso de cambio global hasta el día de hoy (Guzmán et al., 2000: 59).

Frente a este modelo, las décadas de 1960 y 1970 vieron surgir un número creciente y diverso de iniciativas agrarias que proponían alternativas respetuosas con los ciclos ecológicos del suelo. Aunque algunas hundían sus raíces en experiencias de principios del siglo XX, extraían su vigor de la crítica y la sensibilidad ambientales contemporáneas (ibid.: 63). Estas iniciativas fueron agrupándose progresivamente bajo la etiqueta de "agricultura ecológica" y, al mismo tiempo, convergiendo con el nuevo discurso de la sostenibilidad en vista de su carácter propositivo (Caradonna, 2014: 159; Thompson, 1995: 146).

Cada vez más, "agricultura ecológica" se entendía en el imaginario social como la respuesta deseable, sostenible, a los problemas, agroambientales en este caso. Una vez que la sostenibilidad alcanzó el nivel de normalización, institucionalización y consenso (Santamarina, 2006) característico de los anhelos sociales, la agricultura ecológica, de alguna forma, la siguió. La premisa que de ello se deriva es que, en nuestra realidad social, el significante "agricultura ecológica" se ha convertido en la (difusa) denominación que evoca la alternativa deseable respecto de la agricultura industrializada, insostenible. Y ello podría explicar el hecho de que "agricultura ecológica" sea la etiqueta que más usualmente se coloque a toda modalidad agraria o práctica propuesta como alternativa (sostenible) a esa agricultura "convencional".

\section{A MODO DE CONCLUSIÓN}

En este artículo se ha intentado poner las bases para la apertura del foco antropológico en relación a la agricultura ecológica más allá de su existencia como modalidad de manejo agrario. En concreto, se ha explorado un segundo sentido que relaciona a la agricultura ecológica con las formaciones discursivas ambientales. Se ha pretendido así localizar culturalmente la idea de agricultura ecológica y, por ese camino, se ha apuntado hacia la existencia de un estrecho lazo semántico con la sostenibilidad en cuanto "anhelo social" (Žižek, 1997/2003). Se ha argumentado, en este sentido, que la idea de agricultura ecológica constituye la traducción específica al ámbito agrario de ese anhelo, al cual dio lugar el terremoto que significó la irrupción de la crítica ecologista en la década de 1960.

A modo de conclusión del artículo, sólo pretendo desincentivar la posibilidad de que las implicaciones etnográficas que a partir de ahí se puedan desarrollar tengan que ver con un abordaje segregador que descomponga la agricultura ecológica en dos (o más) 
objetos de estudio desconectados o directamente incompatibles. En la viñeta del apartado 3 tenemos un buen ejemplo de la imbricación entre sentidos (y de la dificultad de su deslinde) a un nivel microsocial. La invitación, por tanto, iría más bien en el sentido de profundizar en lo que podría denominarse, parafraseando a Appadurai (1986/1991), la "vida social de la agricultura ecológica", entendida como las formas diversas que adopta la circulación y adopción locales de las prácticas y elementos discursivos que componen la polisemia del término "agricultura ecológica"

En este sentido, es interesante no perder de vista, como proponen Arce y Long (2000) elaborando sobre la visión de la modernidad de Elias, que la extensión en contextos locales de nuevos discursos dominantes y los conjuntos de prácticas que con ellos se imbrican no se desarrolla sin transformaciones. Se producen apropiaciones singulares en función de los actores concernidos, que implican la acomodación en el seno de los universos locales. Desde esta óptica, quizás entendamos mejor la necesidad de abordar objetos de estudio antropológicos como la agricultura ecológica desde la premisa de su incorporación a ensamblajes elásticos y heterogéneos de elementos discursivos y prácticas en continuo proceso de reposicionamiento local. Creo firmemente que la labor del o la antropóloga no es otra que la de abrirse a la complejidad del abajo desde abajo. 


\section{REFERENCIAS BIBLIOGRÁFICAS}

Appadurai, Arjun (1991) "Las mercancías y la política del valor". En Arjun Appadurai (Ed.), La vida social de las cosas. Perspectiva cultural de las mercancías. México D. F.: Grijalbo, pp. 17-87. Publicado originalmente en 1986.

Arce, Alberto y Long, Norman (2000) "Reconfiguring modernity and development from an anthropological perspective". En Alberto Arce y Norman Long (Eds.), Anthropology, development and modernities. Exploring discourses, counter-tendencies and violence. Londres y Nueva York: Routledge, pp. 1-30.

Buck, D., Getz, C. y Guthman, J. (1997) "From farm to table: The organic vegetable commodity chain of Northern California”. Sociologia Ruralis 37(1), pp. 3-20.

Campbell, Hugh y Liepins, Ruth (2001) "Naming organics: Understanding organic standards in New Zealand as a discursive field”. Sociologia Ruralis 41(1), pp. 21-39.

Caradonna, Jeremy (2014) Sustainability. A history. Nueva York: Oxford University Press. Cariño, Micheline y Castorena, Lorella (2016) "Prólogo". En Micheline Cariño y Lorella Castorena (Eds.), Saberes para la sustentabilidad. Barcelona: Icaria, pp. 9-17.

Coca, Agustín (2008) Los camperos. Territorios, usos sociales y percepciones en un "espacio natural" andaluz. Sevilla: Fundación Blas Infante.

Constance, Douglas (2018) "Contested sustainability discourses in the agrifood system: an overview". En D. Constance, J. Konefal y M. Hatanaka (Eds.), Contested sustainability. Discourses in the agrifood system. Abingdon y Nueva York: Routledge, pp. 3-16.

Cortés, José Antonio (2012) Naturalezas en conflicto. Conservación ambiental y enfrentamiento social en el Parque Natural Cabo de Gata-Nijar. Valencia: Germania Associació Valenciana d'Antropologia.

Cruzada, Santiago (2017) "Nosotros también somos indígenas: la vulnerabilidad del naturalismo en contextos occidentales de convivencia entre especies". Etnográfica 21(1), pp. $49-71$.

Dankers, Cora (2004) Las normas sociales y ambientales, la certificación y el etiquetado de cultivos comerciales. Roma: FAO.

Del Campo, Alberto (2000) Agricultores y ganaderos ecológicos en Andalucía. Sevilla: Consejería de Agricultura y Pesca.

Descola, Philippe (2001) "Construyendo naturalezas. Ecología simbólica y práctica social”. En Philippe Descola y Gísli Pálsson (Eds.), Naturaleza y sociedad. Perspectivas antropológicas. México D. F.: Siglo XXI, pp. 101-123. Publicado originalmente en 1996.

Fox, Katy (2011) Peasants into European farmers? EU integration in the Carpathian mountains in Romania. Zúrich y Berlín: Lit Verlag. 
Fournier, Stéphane y Muchnik, José (2012) “El enfoque 'SIAL’ (sistemas agroalimentarios localizados) y la activación de recursos localizados”. Agroalimentaria 34, pp. 133-144.

Galvin, Shaila (2014) "Organic designs and agrarian practice in Uttarakhand, India". Culture, Agriculture, Food and Environment 36(2), pp. 118-128.

Galvin, Shaila (2018) “The farming of trust: Organic certification and the limits of transparency in Uttarakhand, India”. American Ethnologist 45(4), pp. 495-507.

González de Molina, Manuel y Guzmán, Gloria (2006) Tras lospasos dela insustentabilidad. Agricultura y medio ambiente en perspectiva histórica (siglos XVIII-XX). Barcelona: Icaria.

Guthman, Julie (2004) Agrarian dreams: the paradox of organic farming in California. Berkeley y Los Ángeles: University of California Press.

Guthman, Julie (2007) "The Polanyan way? Voluntary food labels as neoliberal governance". Antipode 39(3), pp. 456-478.

Guthman, Julie (2008) "Neoliberalism and the making of food politics in California". Geoforum 39(3), pp. 1171-1183.

Guzmán, G., González de Molina, M. y Sevilla, E. (2000) Introducción a la agroecología como desarrollo rural sostenible. Madrid: Mundi-Prensa.

Hetherington, Kregg (2005) Cultivating utopia: Organic farmers in a conventional landscape. Halifax: Fernwood.

Lozano, Carmen (2011) El sabor de la naturaleza. Agricultura ecológica en parques naturales andaluces. Sevilla: Fundación Blas Infante.

Lozano, Carmen (2013) "Representaciones sociales de la agricultura ecológica en Andalucía”. Gazeta de Antropología 29(2), s. p. Recuperado el 25/01/2016 de http://www. gazeta-antropologia.es/wp-content/uploads/GA-29-2-05-Carmen-Lozano.pdf

Luke, Timothy (1999) "Environmentality as green governmentality". En Éric Darier (Ed.), Discourses of the Environment. Oxford: Blackwell, pp. 21-151.

Martín, Enrique (2014) "Mentiras, inconsistencias y ambivalencias. Teoría de la acción y análisis de discurso”. Revista Internacional de Sociología 72(1), pp. 115-138.

Martínez, Ernesto (2020) “Los acentos del medio ambiente: procesos de ambientalización, fuerza social y articulación discursiva en la Sierra Morena andaluza”. Etnográfica 24(1), pp. 27-46.

Naredo, José Manuel (2004) “Sobre el origen, el uso y el contenido del término sostenible”. Cuadernos de investigación urbanística 41, pp. 7-18. Publicado originalmente en 1997.

Santamarina, Beatriz (2006) Ecología y poder. El discurso medioambiental como mercancía. Madrid: Catarata. 
Santamarina, B., Vaccaro, I. y Beltra, O. (2015) "The sterilization of eco-criticism: From sustainable development to green capitalism". Anduli. Revista andaluza de Ciencias Sociales 14, pp. 13-28.

Schlatter, B., Trávníček, J., Lernoud, J. y Willer, H. (2020) "Organic agriculture worldwide: Current statistics". En FiBL - IFOAM, The world of organic agriculture. Statistics and emerging trends 2020. Frick y Bonn: FiBL - IFOAM, pp. 31-136.

Schmid, Otto (2007) "Development of standards for organic farming". En William Lockeretz (Ed.), Organic farming. An international history. Wallingford y Cambridge: CAB International, pp. 152-174.

Scott, James C. (1998) Seeing like a state: How certain schemes to improve the human condition have failed. New Haven: Yale University Press.

Stavrakakis, Yannis (2000) "On the emergence of green ideology: The dislocation factor in green politics". En D. Howarth, A. Norval y Y. Stavrakakis (Eds.), Discourse theory and political analysis: Identities, hegemonies and social change. Manchester: Manchester University Press, pp. 100-118.

Thompson, Paul (1995) The spirit of the soil. Agriculture and environmental ethics. Londres: Routledge.

Toledo, Víctor (2015) “De qué hablamos cuando hablamos de sustentabilidad? Una propuesta ecológico política”. Interdisciplina 3(7), pp. 35-55.

Vehkamäki, Seppo (2005) "The concept of sustainability in modern times". En Anneli Jalkanen y Pekka Nygren (Eds.), Sustainable use of renewable natural resources: from principles to practices. Helsinki: University of Helsinki, pp. 23-35.

Vogt, Gunter (2007) “The origins of organic farming”. En William Lockeretz (Ed.), Organic farming. An international history. Wallingford y Cambridge: CAB International, pp. 9-29.

Warde, Paul (2018) The invention of sustainability: Nature and destiny, c. 1500-1870. Cambridge: Cambridge University Press.

West, Paige (2010) "Making the market: Specialty coffee, generational pitches, and Papua New Guinea”. Antipode 42(3), pp. 690-718.

Žižek, Slavoj (2003) "Multiculturalismo, o la lógica cultural del capitalismo multinacional”. En Fredric Jameson y Slavoj Žižek, Estudios Culturales. Reflexiones sobre el multiculturalismo ( $2^{\text {a }}$ reimpresión de la $1^{\text {a }}$ ed. en castellano). Buenos Aires: Paidós, pp. 137-188. Publicado originalmente en 1997. 